816 IMPLICATIONS OF THE COVID-19 PANDEMIC: RESULTS OF A GERMAN SURVEY ON PATIENT CARE AND CLINICAL TRIALS IN GYNECOLOGICAL ONCOLOGY (MONITOR-17 SURVEY)

'S Nasser*, ${ }^{2} \mathrm{D}$ Zocholl, ${ }^{2} \mathrm{~S}$ Boz, ${ }^{2} \mathrm{M}$ Keller, ${ }^{1} \mathrm{D}$ Dimitrova, ${ }^{1} \mathrm{R}$ Armbrust, ${ }^{1} \mathrm{JU}$ Blohmer, ${ }^{3} \mathrm{C}$ Fotopoulou, ${ }^{4,5} \mathrm{~A}$ Dubois, ${ }^{1} \mathrm{MG}$ Inci, ${ }^{1} \mathrm{~J}$ Sehouli. ${ }^{1}$ Charite Comprehensive Cancer Center, Berlin, Germany; ${ }^{2}$ North-East German Society for Gynecological Oncology, Germany; ${ }^{3}$ Queen Charlotte's Hospital, Imperial College London, London, UK; ${ }^{4}$ Germany, Germany; ${ }^{5}$ Kliniken Essen-Mitte, Gynecological Oncology, Essen, Germany

\subsection{6/ijgc-2021-ESG0.319}

Introduction/Background* This survey describes the Germanwide impact of the COVID-19 pandemic on provision of clinical care and recruitment in clinical trials of patients with gynecologic malignancies from a physician's perspective.

Methodology We performed an online anonymous multicentric prospective survey across clinicians in Germany. The multiple-choice questionnaire was administered at 4-6 weekly intervals from April 2020 to October 2020 for a total of four series.

Result(s)* 483 questionnaires were completed. The majority of participants were gynecological oncologists $(83.3 \%)$ in certified gynecologic cancer centers (61\%) and breast cancer (BC) centers $(80.4 \%)$. The majority stated a $50 \%$ reduction in surgical interventions for gyne-oncological cases. Cases that were prioritized for surgery across all tumors were those with early stage disease, at primary situation and with a good ECOG status. For BC, patients following neoadjuvant chemotherapy treatment and those with high-risk or locally-advanced BC were prioritized. The majority (73\%) continued to conduct clinical trials throughout the pandemic. In cases were trials were discontinued, this decision was made by sponsors, and hospital officers. Other reasons for discontinuation included lack of patient-participation (due to fear of attending appointments). Almost $100 \%$ of the responders refuted any increased tendency to treat with a neoadjuvant approach (cytotoxic, hormonal, radiation) patients that would qualify for surgery under normal circumstances. This comes in direct contrast to the increased attitude to treat with neoadjuvant anticancer therapy of advanced cancers in other European countries.

Only $18 \%$ of the clinicians reported feeling adequately informed about established safety pathways for COVID-19 positive patients with gynecological cancers. More than $43 \%$ of the clinicians felt that the COVID-19 pandemic will continue to impact on clinical care for up to 2 years.

Conclusion* Targeted emergency algorithms for patients with gynecological cancers need to be developed to protect and preserve care and treatment options for our patients in future pandemics.

\section{COMPLIANCE WITH ENHANCED RECOVERY AFTER SURGERY IN A GYNAECOLOGICAL ONCOLOGY SERVICE IN THE WEST OF IRELAND}

C O'gorman*, E Loughnane, D Zibar, L O'sullivan, SA Azman, K Astbury, M O'leary. University Hospital Galway, Obstetrics and Gynaecology, Galway, Ireland

\subsection{6/ijgc-2021-ESG0.320}

Introduction/Background* Enhanced recovery after surgery (ERAS) protocals aim to improve clinical outcomes and provide a cost benefit to the healthcare system. This approach is widely accepted as the goal in perioperative care, but implementation varies widely. The ERAS Society updated their guidelines for perioperative care in gynecological oncology in early 2019.

Methodology Retrospective review of implementation of ERAS recommendations in a gynaecological oncology service in the west of Ireland. Data collection through review of patient charts for all major surgeries in the service over 4months (September-December 2019 inclusive).

Result(s)* Total cohort of patients undergoing surgery during this time period was 41 women. One cervical cancer, 13 endometrial cancers and 27 tubo-ovarian pathologies. No prehabilitation and verbal education only offered. Successful smoking cessation $(>4 / 52)$ in $50 \%$ of smokers. Bowel preparation used in $2.4 \%$ of patients. Length of time fasting; 68hours (10\%), 8-12hours (46\%), and 12-16hours (44\%), no carbohydrate loading pre-operatively. Compliance was $100 \%$ with anti-microbial prophylaxis, normothermia maintenance, and chlorhexidine based skin preparation at surgery, but no pre-operative chlorhexidine based showers recorded. Peritoneal drains were used in $34 \%$ of cases, and there was no strict maintenance of normoglycaemia. There was $100 \%$ compliance with intra-operative/post-operative dual mechanical/chemothromboprophylaxis, but no pre-operative, nor extended postoperative thromboprophylaxis. General anaesthetic in 100\% cases. No sedatives given pre-operatively and $42 \%$ underwent minimally invasive surgery. At laparotomy, 92\% had a thoracic epidural sited (average use 2.5days), and 12.5\% had patient controlled analgesia. Multi-modal anti-emetics used in $68 \%$ of cases and an nasogastric tube placed in 2.4\%. Postoperative opiates prescribed in 90\%, NSAIDs in 93\%, no use of gabapentin recorded. Oral diet resumed in under 24 hours for $61 \%$, with $39 \%$ resuming diet in the following 24 hour period. Urinary catheters were removed in under 24 hours for $42 \%$ of patients, with $24 \%$ and $34 \%$ in the following 24 hour periods respectively $(71 \%$ of which were related to ongoing epidural use). All patients without epidurals mobilised in under 24 hours. Of those with epidurals, 77\% mobilised between 24-48 hours.

Conclusion* Mixed compliance is demonstrated with ERAS guidance. There is excellent compliance in the area of surgical-site infection prevention. Improved pre-operative education, reduced fasting times, carbohydrate loading, pre- and postoperative extended thromboprophylaxis and reduced opiate use would improve ERAS compliance rates.

\section{AUDIT TO STREAMLINE MULTIDISCIPLINARY TEAM MEETINGS BY IMPLEMENTING STANDARD OF CARE FOR EARLY ENDOMETRIAL CANCER CASES}

P Bansal* , FA Sefre. Northampton General Hospital, Northampton, UK

\subsection{6/ijgc-2021-ESG0.321}

Introduction/Background* Multi-disciplinary team(MDT) meetings are the core component of the UK's cancer services. Over the last decade, incidence rates for all cancers combined have increased by a twentieth $(5 \%)$ in the UK. UK's health services have changed significantly since the introduction of MDT in 1995 and MDT are already under considerable pressure.

In 2020 NHS CANCER ALLIANCES published guidance on how MDTs can streamline to focus time on more complex cases through the introduction of Standards of Care (SoCs) 
Methodology at Northampton general hospital ,we determined the SoCs for early endometrial endometriod cancer (EEC) and uterine sarcomas in accordance to BGCS guidelines. Then conducted an audit, examined the uterine cancer cases discussed in MDTs retrospectively over the period of nov 2020 to march 2021 .Cases which were reviewed are endometrial endometriod cancer (FIGO stage 1) and cases of fibroid uterus discussed due to suspicion of uterine sarcomas. In total 63 EEC and 18 fibroid uterus cases were discussed in 21 MDT meetings over 5 months.

Result(s)* Radiologically and histologically the cases were in concordance to the initial reporting thus not affecting the management decision of EEC . 20\% of EEC and 38\% of fibroid uterus cases were discussed moe than once due to logistical issues. Of Fibroid uterus cases , $84 \%$ turned out to be benign and 15\% malignancies.MDT discussion could not contribute to the sarcomas as radiologically degenerating fibroids vs sarcoma decision was inconclusive leading to standard laparotomy and debulking decision, after ruling out extra uterine disease.

Conclusion* Recommendations drawn of this audit will now be implemented as- Each MDT will have a separate section as not for full discussion list. These cases will only be discussed if there is any doubt, any queries, or new information becomes available. Regular audit will be conducted of cases not discussed in relation to the appropriateness of patients receiving a SoC and their outcome on the following grounds-

Percentage of patients assigned to SoC compared to overall caseload to assess scope of streamlining. How is streamlining impacting on time for those patient cases which require discussion? What is the impact on total length of the MDT.

\section{SURGICAL ONCOLOGY FOR NON-COVID-19 PATIENTS DURING THE PANDEMIC}

${ }^{1} \mathrm{~K}$ Ben Hamida*, ${ }^{1} \mathrm{H}$ Bouaziz, ${ }^{1} \mathrm{~A}$ Jellali, ${ }^{1} \mathrm{~N}$ Tounsi, ${ }^{1} \mathrm{H}$ Bouzaiene, ${ }^{2} \mathrm{M}$ Hsairi, ${ }^{1} \mathrm{M}$ Slimane, ${ }^{1} \mathrm{~K}$ Rahal. 'Salah Azaiez Institute of Oncology, Department of Surgical Oncology, Tunis, Tunisia; ${ }^{2}$ Salah Azaiez Institute of Oncology, Department of Epidemiology and Medical Statistics, Tunisia

\subsection{6/ijgc-2021-ESG0.322}

Introduction/Background* The COVID-19 pandemic has turned the standard of care of medicine worldwide into a "public health emergency of international concern. "

Cancer patients are a unique population in that they are vulnerable to COVID-19, particularly if immunocompromised, and also, their oncologic outcome is based on the type and timing of the treatment.

Methodology A retrospective review comparing all surgical activities between the year before COVID-19 and the year after.

This study was conducted in the Surgical Oncology Department in Salah Azaiez Institute of Oncology, the reference cancer care center in Tunisia.

Result(s)* In our center, we created a new surgical procedures team.

There was a significant reduction in the median daily breast surgery, superficial surgery, and ambulatory surgery $(<0,001)$. But not all operation types decreasing in frequency.

Three months after COVID -19, we selected patients for laparotomy and decreased our activity by $50 \%$.
Starting from June 2020, we have increased our activity to exceed that of 2019. No increase in mortality or morbidity from treatment during COVID-19 for operated patients.

The total surgically consults volume decreased by $36,17 \%$ in the post-COVID-19 period, significantly reducing the median daily consult volume $(\mathrm{p}<0.001)$.

Conclusion* In this new context, the decision for surgery is driven not only by what is best for the individual patient but also by the concern for transmitting COVID-19 to patients and health care workers.

\section{THE IMPACT OF COVID-19 PANDEMIC ON GYNECOLOGICAL AND BREAST CANCER DETECTION RATE: A TERTIARY CENTER PERSPECTIVE}

K Knoll*, E Reiser, I Tsibulak, K Leitner, J Kögl, C Ebner, C Marth. Medical University of Innsbruck

\subsection{6/ijgc-2021-ESG0.323}

Introduction/Background* The aim of the present study was to assess the impact of postponed screening examinations and lockdown measures on gynecological and breast cancer detection rate throughout the year 2020 in a gynecological oncological center in Austria.

Methodology Data of 889 patients with either newly diagnosed gynecological or breast cancer between January 2019 and December 2020 were collected. Clinical parameters including symptoms, performance status, comorbidities and referral status were compared in patients, who were newly diagnosed with cancer in the period of the first lockdown from March 2020 - April 2020 and the second lockdown from November 2020 - December 2020 and compared to the same period in 2019 .

Result(s)* Our results showed a strong decline in newly diagnosed cancers during the lockdown periods: $-45 \%$ in gynecological cancer and $-52 \%$ in breast cancer compared to the same period in 2019. Compared to the analogue period of 2019, breast cancer patients reported significantly more tumor-associated symptoms (55\% versus 31\%, $\mathrm{p}=0.013)$ during and in between ( $48 \%$ versus $32 \%, \mathrm{p}=0.022)$ the lockdowns. During the lockdown periods breast cancer patients were diagnosed with a significantly higher tumor-stage (T2-T4; $\mathrm{p}=0.047)$.

Conclusion* Both lockdowns led to a strong decrease in newly diagnosed gynecological and breast cancers. Treatment delays in potentially curable disease could lead to inferior clinical outcomes, with the risk of missing the optimal treatment window. As the COVID-19 pandemic will be a challenge for some time to come, new strategies in patient care are needed to optimize cancer screening and management during the pandemic.

\section{INTENT OF TREATMENT IN MAJOR GYNAE-ONCOLOGY SURGERY - A QUALITY IMPROVEMENT PROJECT IN A TERTIARY TEACHING HOSPITAL}

H Asif*, T Nomani. Royal Wolverhampton NHS Trust, Obstetrics and Gynaecology, Wolverhampton, UK

10.1136/ijgc-2021-ESG0.324 